\title{
ArcheoSciences
}

Revue d'archéométrie

\section{Dosages par fluorescence X portable d'ateliers médiévaux de production des métaux non-ferreux}

Les exemples de Castel-Minier et d'Agnesserre (Aulus-les-bains, Ariège)

Survey of non-ferrous medieval workshops by field-portable XRF soil analysis at Castel-Minier and Agnesserre (Aulus-les-Bains, Ariège)

Florian Téreygeol, A. Arles, E. Foy, Nicolas Florsch et M. Llubes

\section{(2) OpenEdition}

\section{Journals}

\section{Édition électronique}

URL : https://journals.openedition.org/archeosciences/2802

DOI : $10.4000 /$ archeosciences.2802

ISSN : 2104-3728

Éditeur

Presses universitaires de Rennes

Édition imprimée

Date de publication : 10 avril 2010

Pagination : 243-252

ISBN : 978-2-7535-1407-2

ISSN : $1960-1360$

\section{Référence électronique}

Florian Téreygeol, A. Arles, E. Foy, Nicolas Florsch et M. Llubes, « Dosages par fluorescence X portable d'ateliers médiévaux de production des métaux non-ferreux », ArcheoSciences [En ligne], 34 | 2010, mis en ligne le 11 avril 2013, consulté le 31 janvier 2022. URL : http://journals.openedition.org/ archeosciences/2802; DOI : https://doi.org/10.4000/archeosciences.2802

Ce document a été généré automatiquement le 31 janvier 2022.

Article L.111-1 du Code de la propriété intellectuelle. 


\title{
Dosages par fluorescence X portable d'ateliers médiévaux de production des métaux non-ferreux
}

\author{
Les exemples de Castel-Minier et d'Agnesserre (Aulus-les-bains, Ariège) \\ Survey of non-ferrous medieval workshops by field-portable XRF soil analysis at \\ Castel-Minier and Agnesserre (Aulus-les-Bains, Ariège)
}

Florian Téreygeol, A. Arles, E. Foy, Nicolas Florsch et M. Llubes

\section{Introduction}

1 Le problème lié à la prospection des sites de métallurgies des non-ferreux se pose de façon cruciale pour l'archéologue. À la différence des ateliers de production du fer, l'empreinte topographique laissée par cette activité humaine est bien moindre. Pour s'en persuader, il suffit de dénombrer les découvertes des ateliers des deux types. À l'inverse, il est indéniable que, au moins pour l'Europe médiévale, les métaux les plus recherchés par les anciennes sociétés restent l'or et l'argent. Il serait facile, et un peu rapide, de relier ces différences quantitatives uniquement à la prédominance naturelle $\mathrm{du}$ fer dans la nature et à son usage commun. La perception naturaliste de cette variation doit être pondérée par la soif des métaux nobles. Dès lors, s'il est plus simple de localiser des unités de production du fer, la raison n'en incombe pas uniquement à la surabondance de ce minerai. D'autres causes peuvent être évoquées. Une cause conjoncturelle tout d'abord. Il est un fait en France que le nombre des archéologues travaillant sur le fer est plus important que celui des archéologues travaillant sur les métallurgies non-ferreuses. Une raison structurelle déjà mentionnée mais qui doit être explicitée : les déchets de la métallurgie du fer sont bien plus prégnants dans le paysage que ceux des non-ferreux. Non seulement les scories issues des chaînes opératoires des métallurgies des non-ferreux connaissent systématiquement un tri et un concassage pour récupérer le métal piégé dans le verre. Mais le résidu de ce retraitement peut connaitre une seconde vie soit comme fondant réinjecté dans la même chaîne 
opératoire dont il est issu, soit comme matière première dans d'autres systèmes de production (Téreygeol et al., 2004). Et enfin une dernière raison, structurelle là encore. Comme l'ont montré pour le fer Claude Domergue et Marc Leroy, il existe un réel changement d'échelle dans la production métallique entre l'antiquité et la période médiévale (Domergue et Leroy, 2000). L'Europe médiévale occidentale ne connait pas d'équivalent à des sites antiques, comme ceux de Rio Tinto ou des Martys. Même des grands districts comme Kutna Hora, l'Erzgebirge ou Sainte-Marie-aux-Mines n'ont pas laissé de traces évidentes de la métallurgie qui s'y est déroulé durant plusieurs siècles. Fort de ce constat, comment espérer repérer des ateliers de production de plomb et d'argent? Nous disposions pour cela de l'arsenal classique dévolu à la prospection archéologique allant de la prospection pédestre jusqu'à la télédétection satellitale. Mais des problèmes demeurent reposant tant sur le coût des méthodes, que sur leur difficulté de mise en œuvre, ou encore leur pertinence. Avec la miniaturisation des appareils d'analyses et le réel développement de leur caractère portable, il devient possible de repenser une des approches prospectives des ateliers de production des métaux non-ferreux : l'étude géochimique des sols.

\section{1. État de l'art}

2 Dans un premier temps, c'est pour des problématiques géologiques et environnementales que le caractère portable des appareils de dosage a été développé. En l'état actuel des recherches, c'est l'analyse par spectrométrie de fluorescence $\mathrm{X}$ qui est très généralement employée car elle offre de nombreux avantages. C'est d'abord une technique de détermination non invasive, ni destructive. La simplicité du phénomène physique mis en jeu permet d'obtenir rapidement des informations concernant la composition d'un échantillon. L'approche qualitative est fiable. En revanche, l'obtention d'une donnée quantitative implique des calibrations préalables.

3 Le principe de la méthode décompose les appareillages les plus courants en trois grands organes qui ont fait l'objet d'améliorations indépendantes : une source de production de rayons $\mathrm{X}$ excitatrice à laquelle sont soumis les échantillons, un détecteur qui permet de mesurer la désexcitation de fluorescence, propre aux éléments analysés, ainsi qu'un analyseur de ce signal. C'est sur les deux premiers appareillages que portent les recherches les plus récentes, afin d'en proposer des miniaturisations. Quant aux améliorations apportées au troisième composant, plus que de gagner en portabilité, elles sont dirigées dans un sens d'optimisation de la qualité des analyses.

4 La première étape vers la conception d'appareils portables prend place au début des années 90 avec le développement de microprocesseurs puissants permettant de traiter les signaux produits. L'utilisation d'un système de refroidissement électronique par effet Pelletier ${ }^{1}$ a réellement ouvert la voie à la portabilité en permettant l'abandon des encombrants réservoirs d'azote. Les améliorations récentes ont ensuite été apportées à la technologie des sources de rayonnements $\mathrm{X}$. Le remplacement des sources radioactives au profit de tubes de rayon $\mathrm{X}$ basse tension a non seulement permis l'usage de l'appareil sur batterie mais a également diminué le risque radiologique.

Aujourd'hui, il existe sur le marché des spectromètres de fluorescence $\mathrm{X}$ dont la masse est inférieure à deux kilogrammes, développés spécialement pour une utilisation hors laboratoire. Les applications industrielles concernent trois grands domaines: la production minière, le contrôle qualité et la gestion du risque sanitaire. L'archéométrie 
est aussi un domaine, certes plus réduit, qui a bénéficié de cette miniaturisation pour faciliter les caractérisations au sein des musées, d'objets archéologiques jusqu'alors jamais analysés car trop précieux, fragiles ou volumineux pour être déplacés vers un laboratoire. Cependant, les appareils portables de spectrométrie utilisés dans la majorité des études publiées dans ce domaine ne sont pas véritablement des instruments de terrain à la différence de ceux employés dans l'industrie. Il s'agit plutôt d'instrumentations transportables ${ }^{2}$ souvent qualifiées de laboratoires mobiles et, de fait, incompatibles avec un fonctionnement sur un site archéologique. En archéologie, l'utilisation d'appareils portables d'analyse par spectrométrie de fluorescence $\mathrm{X}$ a été proposée pour des caractérisations directement sur le terrain dans un but d'aide à l'échantillonnage et à la compréhension des différentes phases d'activités. Dans le cadre de prospections réalisées sur des sites paléométallurgiques, la mise en évidence de multiples classes de composition de scories permet de définir des exploitations ou des technologies distinctes (Wagner et al., 1983).

6 La démarche prospective qui sera définie par la suite, est assimilable à celles que l'on retrouve en exploration minière ou dans le cadre de caractérisation de sites pollués. Elle repose sur une approche géochimique extensive d'un site archéologique. Il faut considérer qu'une activité métallurgique ancienne, en tant qu'industrie polluante, aura laissé dans le sol des résidus de son fonctionnement. La localisation de sources de pollutions dans une zone de prospection doit permettre de localiser les vestiges archéologiques potentiels enfouis qui en sont à l'origine. Parallèlement, nous proposerons une autre approche directement sur les couches archéologiques et sur les stratifications permettant d'améliorer la compréhension du séquençage des activités sur un site.

7 La considération de données géochimiques en archéologie n'est pas une démarche inédite. Elle se définit par la recherche dans les sols d'une zone d'intérêt d'anomalies de composition résultant d'une activité anthropique. Les premières études publiées décrivant ce type de prospection révélaient l'occupation humaine d'un espace par une augmentation du taux de phosphore dans le sol. C'est August Arrhenius qui, au cours de ses travaux visant à réaliser des cartographies de composition de sol pour une entreprise sucrière suédoise, remarque le premier une augmentation du taux de phosphore dans les zones à proximité de sites archéologiques d'occupation médiévale (Arrhenius, 1931).

8 Dans le domaine de l'archéologie minière et métallurgique, plusieurs auteurs exposent une démarche voisine à celle qui vient d'être décrite (Moraz et Pulou, 1997). Également dans le cadre d'études archéologiques de sites de production de l'argent, certains abordent des problématiques liées aux périodes de fonctionnement de ces activités d'un point de vue géochimique différent. À partir d'études de carottes prélevées dans les tourbières ou les glaciers, il est possible par une approche multidisciplinaire, de dater et d'évaluer la durée de fonctionnement d'activités métallurgiques mais à des échelles qui dépassent généralement celle d'un site tant dans la dimension spatiale que temporelle (Nieminen et al., 2002 ; Ploquin et al., 2003).

Enfin, un dernier aspect de l'utilisation de données géochimiques en archéologie se rapproche de celui que nous avons mis en œuvre. Il s'agit de méthodes appliquées à la recherche de sites archéologiques de métallurgie des métaux non-ferreux qui vont chercher à mettre en évidence dans les sols, des concentrations anormales d'éléments métalliques. Elles supposent que ces anomalies géochimiques témoignent a priori 
d'activités anthropiques. On y reconnaitt ici une démarche similaire à celle décrite pour les problématiques liées à la détection du phosphore. Dans les sédiments d'une zone expertisée, la mise en évidence de fortes concentrations en métaux lourds doit permettre de proposer la localisation des lieux où s'établissaient les opérations à l'origine des pollutions constatées. Dans l'article de Marie-Christine Bailly-Maître et Nicolas Jacob, la mise en place de cette méthode apparaît limitée par la technique analytique choisie qui oblige un retour au laboratoire, introduisant un décalage temporel entre les observations de terrain et les résultats analytiques intervenant $a$ posteriori (Bailly-Maître et Jacob, 2001). À l'inverse, l'emploie d'appareils d'analyse par spectrométrie de fluorescence $\mathrm{X}$ décrit dans deux récentes publications semble faciliter la compréhension in situ d'un site archéologique orientant instantanément une prospection (Shalev et al., 2006 ; Berna et al., 2007).

\section{La méthode envisagée : le dosage par fluorescence $\mathrm{X}$ portable}

10 Le travail présenté ici a été conduit entre 2006 et 2007 à l'aide d'un spectromètre de fluorescence X (XRF) portable (Fondis, Niton XLt). Celui-ci est spécialement conçu pour l'analyse des métaux et alliages sous forme solide, des minerais et des sols. C'est cette dernière fonctionnalité qui a été mise en œuvre. Les tension et intensité maximales du tube sont respectivement de $35 \mathrm{kV}$ et $10 \mu \mathrm{A}$ délivrant une puissance de $1,7 \mathrm{~W}$. Le tube générateur de rayons $\mathrm{X}$ est refroidi par air, son anode est en argent avec une fenêtre de sortie en béryllium de $0,127 \mathrm{~mm}$ d'épaisseur. La section du faisceau (spot) à l'endroit où il touche l'échantillon est approximativement de $20 \mathrm{~mm}$ par $10 \mathrm{~mm}^{3}$. Les éléments qu'il est possible de détecter vont du potassium $(\mathrm{K}, 19)$ au plutonium $(\mathrm{Pu}, 94)$. Dans le cas de notre étude, la durée de l'analyse est de 30 secondes par échantillon. Des durées plus longues ont été testées sans apporter d'amélioration notable par rapport à nos besoins.

11 Le résultat est donné en ppm. Des calibrations ont été effectuées par analyse par ANRC au Centre Ernest Babelon ${ }^{4}$ pour l'étain, le zinc, l'antimoine, le plomb, l'argent, l'arsenic et le cuivre, et par ICP-MS au CRPG de Nancy pour plus de 55 éléments ${ }^{5}$. Les résultats sont particulièrement positifs et permettent d'utiliser directement les teneurs en plomb, zinc et cuivre obtenues par XRF portable. D'autre part, l'expérience nous a montré que les analyses étaient reproductibles, que nous n'avions pas d'effet de pépite pour les autres éléments comme le fer ou l'arsenic, et que la quantification avait une logique intrinsèquement à notre prospection; logique que nous démontrons à l'aide des données proprement historiques et archéologiques.

Nous avons mis en place des approches complémentaires: une à but strictement prospectif, l'autre visant à mieux comprendre le terrain en cours de fouille. De la même façon, deux modes de collecte d'informations ont été définis. Dans le cadre prospectif, les échantillons sont prélevés à la tarière à $30 \mathrm{~cm}$ de profondeur. Le maillage de prélèvement dépend de la surface à couvrir et de la connaissance préalable du site. Pour Castel-Minier, la zone d'intérêt couvre deux hectares et les informations tant archéologiques qu'historiques dont nous disposons sont nombreuses. La prospection s'y est faite à maille fine (un prélèvement tout les $10 \mathrm{~m}$ ). À l'inverse sur le site d'Agnesserre, nos données sont beaucoup plus ténues et l'espace à couvrir avoisine les 5 hectares. Après une étude géophysique, le choix d'échantillonnage s'est orienté vers une approche extensive se calquant sur la méthode de prospection par stream sediments 
appliquée en prospection minière. Une fois recueillis, les échantillons sont ramenés à notre "laboratoire de campagne" pour être tamisés à une maille de $2 \mathrm{~mm}$. Ils sont ensuite mis en capsule et recouverts d'un film Mylar $(6 \mu \mathrm{m}$, diamètre. $63,5 \mathrm{~mm})$ et scellés. À l'inverse, lors de l'étude des coupes stratigraphiques, le dosage s'effectue directement in situ sans prélèvement, ni préparation d'échantillon. La réponse pourrait sembler alors de moindre qualité et pourtant des comparatifs établis à l'occasion d'une autre prospection ont validé cette stratégie de collecte d'informations (Téreygeol et al., 2007). L'objectif n'est pas de fournir une composition globale des sols mais bien de réaliser une cartographie à partir des métaux lourds provenant de l'activité minière à savoir le plomb, le zinc et le cuivre. L'usage dans cet appareil d'une anode en argent ne permet pas de discriminer l'argent de l'échantillon, de celui de la machine. Nous notons également le dosage du fer bien que la calibration de l'appareil ne soit pas satisfaisante pour cet élément. En effet, le site de Castel-Minier est également le lieu d'implantation d'une mouline, appareil sidérurgique profitant des installations hydrauliques nécessaires à la bonne exploitation de la mine.

\section{Prospections et résultats}

Le site choisi pour être sujet de cette prospection géochimique est celui de Castel-Minier en Ariège (fig. 1). Il s'agit d'un ensemble composé qui associe à une mine d'argent exploitée entre les $\mathrm{XII}^{\mathrm{e}}$ et $\mathrm{xIV}^{\mathrm{e}}$ siècles au lieu-dit Les Ouels, un ensemble castral, aujourd'hui rasé, et des vestiges d'activités minéralurgiques aussi bien à Castel-Minier que dans le cirque d'Agnesserre. Moins visibles, les espaces métallurgiques sont néanmoins présents. Les fouilles en cours ont permis de caractériser l'existence d'une mouline (XIII $-1^{\text {re }}$ moitié du XVI ${ }^{\mathrm{e}}$ siècles), un atelier de production de fer, alors que les traces de la métallurgie de l'argent se limitent à quelques objets dont des scories et un lingot de plomb. En revanche, les textes éclairent quasi exclusivement les questions liées à la production du métal blanc (par exemple : Leroy, 1972). 
Figure 1 : Carte de localisation.

Figure 1: Map showing the site location.

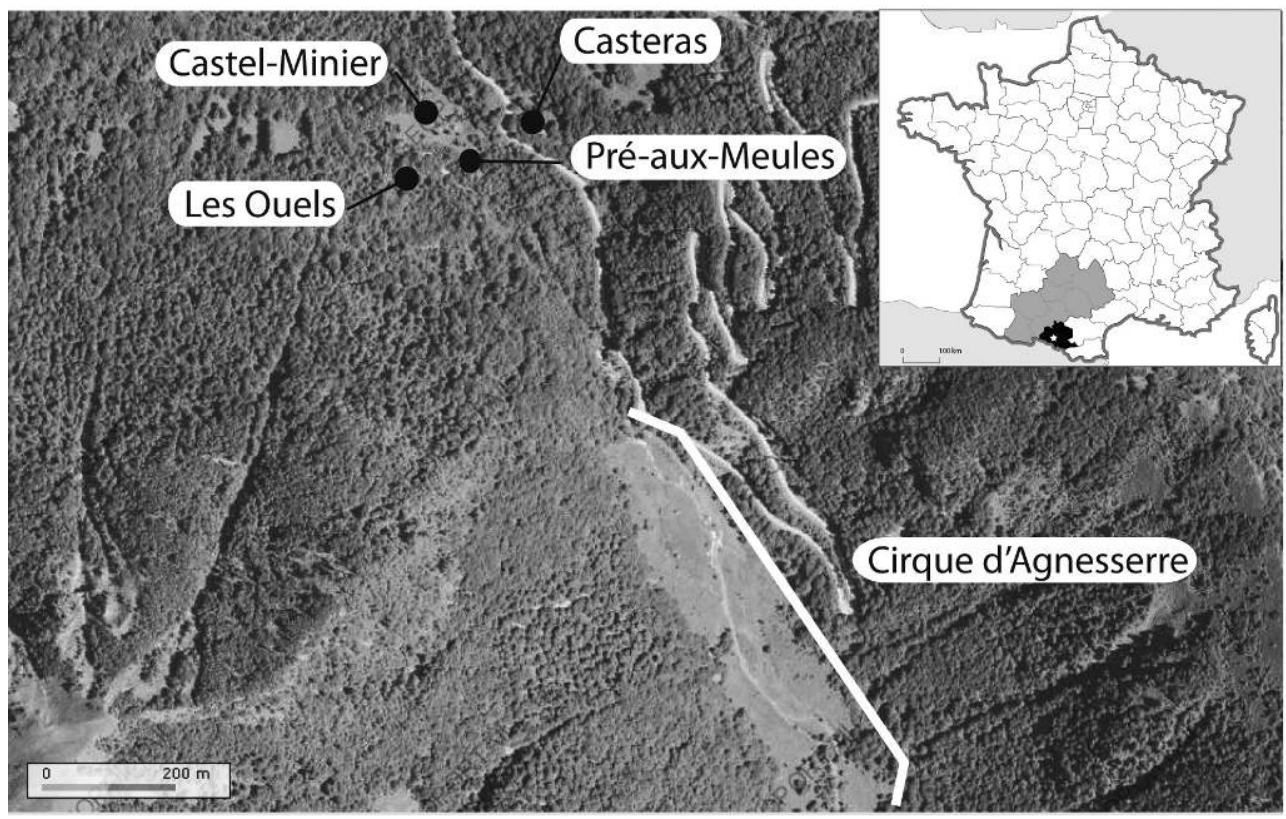

\section{Définition du fond géochimique}

14 Ce travail de prospection prend place dans un espace complexe en termes d'étude géochimique dans la mesure où nous sommes confrontés à des sols contenant des métaux lourds d'origine naturelle autochtone (érosion de roches, lessivage), une part toujours naturelle mais allochtone représentative des matières premières importées et préparées sur le site (même si le déplacement se limite au franchissement du torrent) et une fraction anthropique résultant de la transformation des minerais dans le cadre des opérations métallurgiques. De par les teneurs recherchées et l'emplacement du site, nous avons pu faire abstraction des pollutions modernes. Des dosages ayant valeur de «blanc » ont été réalisés pour fournir un niveau de seuil représentatif de la fraction naturelle autochtone. Ces dosages ont été réalisés sur le versant nord du Garbet, audessus du site de Castel-Minier, au lieu-dit Castéras. Ils fournissent une moyenne de 500 ppm de plomb, 300 ppm de zinc, $3 \%$ de fer alors que le cuivre reste sous le seuil de détection (moins de $30 \mathrm{ppm}$ ).

Une série d'analyses a été faite également sur la rive gauche où se trouvent les mines afin de définir le fond géochimique sur le gisement après son exploitation. L'interprétation est plus complexe puisque les sols se placent directement sur une zone minéralisée avec des dépôts de déchets miniers, donc, a priori, enrichie naturellement en métaux lourds. La présence des métaux varie assez fortement d'une zone de prélèvement à l'autre (fig. 2). Il y a une bonne corrélation entre le plomb, le zinc et le cuivre en accord avec la nature du gisement qui est de type plombo-zincifère. Les plus fortes concentrations de zinc mais également de plomb se localisent sur les haldes car, au Moyen âge, la sphalérite n'était pas exploitée par les mineurs et son rejet a entrainé quelques pertes en galène. 
Figure 2 : Résultat des blancs pris sur le versant minier (Les Ouels). Figure 2: Geochemical background on the mining site (Les Ouels).

\begin{tabular}{|c|c|c|c|c|c|c|c|}
\hline Nature du sous-sol & $\begin{array}{c}\text { Zone } \\
\text { filonienne }\end{array}$ & $\begin{array}{c}\text { Zone } \\
\text { filonienne }\end{array}$ & Halde & Halde & Halde & $\begin{array}{c}\text { Zone } \\
\text { filonienne }\end{array}$ & $\begin{array}{c}\text { Zone } \\
\text { filonienne }\end{array}$ \\
\hline Ref CM & CM 86 & CM 87 & CM 88 & CM 89 & CM 90 & CM 91 & CM 92 \\
\hline Pb en ppm & $\mathbf{8 4 5 0}$ & $\mathbf{7 3 3 0}$ & $\mathbf{1 3 7 0 0}$ & $\mathbf{1 4 2 0 0}$ & $\mathbf{2 6 0 0 0}$ & $\mathbf{7 1 0 0}$ & $\mathbf{1 2 6 0}$ \\
\hline Zn en ppm & 980 & 1380 & 2240 & 3330 & 4510 & 1640 & 1500 \\
\hline Cu en ppm & 153 & 87 & 221 & 213 & 232 & 29 & 13 \\
\hline Fe en mas\% & $3,8 \%$ & $3,3 \%$ & $4,2 \%$ & $4,5 \%$ & $5,4 \%$ & $7 \%$ & $14 \%$ \\
\hline
\end{tabular}

16 À l'aide de ces analyses, nous considérerons que les dosages inférieurs à 500 ppm de plomb, et ceux inférieurs à 300 ppm de zinc s'inscrivent dans un fond naturel sur la zone de Castel-Minier. Au-delà, nous caractérisons un apport anthropique. Ces seuils ne sont valables que pour le versant droit de Castel-Minier et pour Agnesserre. Ils ne peuvent en aucun cas être appliqués à la zone minière (Les Ouels).

\section{Prospections géochimiques extensive et systématique}

17 Dans le cadre de cette approche en prospection extensive, deux sites ont pu être testés sur cette zone. Sur l'un, Castel-Minier, nous disposons déjà d'un lot d'informations tant géophysique, archivistique qu'archéologique permettant à l'issu de la prospection d'affiner les conclusions. Pour le cirque d'Agnesserre, non seulement les connaissances sont beaucoup plus réduites, mais la surface à traiter est plus du double de celle de Castel-Minier.

\section{Le cas de Castel-Minier}

18 Avant d'entamer l'étude géochimique de ce terrain, des prospections géophysiques ont été réalisées (Bonnamour et al., 2007). Certains espaces se sont révélés inaccessibles à cette approche soit pour des raisons topographiques (végétation, espace réduit, etc.) soit pour des raisons inhérentes aux méthodes mises en œuvre (proximité d'éléments métalliques, etc.). La prospection géochimique se posait donc comme un complément appréciable à l'analyse géophysique du site devant guider l'archéologue dans sa stratégie de fouille après une approche systématique du terrain.

19 Les prélèvements ont été effectués avec un espacement de 10 mètres sur des lignes matérialisées par des décamètres. Chaque prélèvement a été localisé à l'aide d'un tachéomètre. Il dispose ainsi de coordonnées en $\mathrm{X}$ et $\mathrm{Y}$, le $\mathrm{Z}$ représentant la quantification d'un élément. Ce système de référencement est bien évidemment calé sur notre propre relevé topographique. Nous sommes à même de restituer une carte des différents éléments dosés drapée sur le fond cartographique.

Les métaux travaillés sur le site sont le plomb, l'argent et le fer. La production médiévale d'argent n'a pas donné lieu à des concentrations que l'on soit à même de doser avec la méthode employée. En revanche, la carte du plomb est édifiante (fig. 3). Elle met en évidence trois niveaux de concentration sur trois zones particulières. En bordure du torrent, la présence de plomb a été notée avec un taux compris entre 5000 et $10000 \mathrm{ppm}$. À l'emplacement de la fortification, la concentration en plomb monte jusqu'à 30000 ppm. Enfin sur le lieu-dit "le pré-aux-meules", les taux de plomb atteignent des sommets avec un zonage à plus de $40000 \mathrm{ppm}$. Lorsque l'on dresse les 
cartes du zinc et du cuivre, l'image est de prime abord la même. Les variations sont à mettre sur le compte des différences de mobilité des éléments dans les sols.

Figure 3 : Cartes de Castel-Minier des teneurs en plomb, cuivre, zinc et fer (en ppm).

Figure 3: Castel-Minier Geochemical maps showing Lead, copper and zinc concentration (ppm).
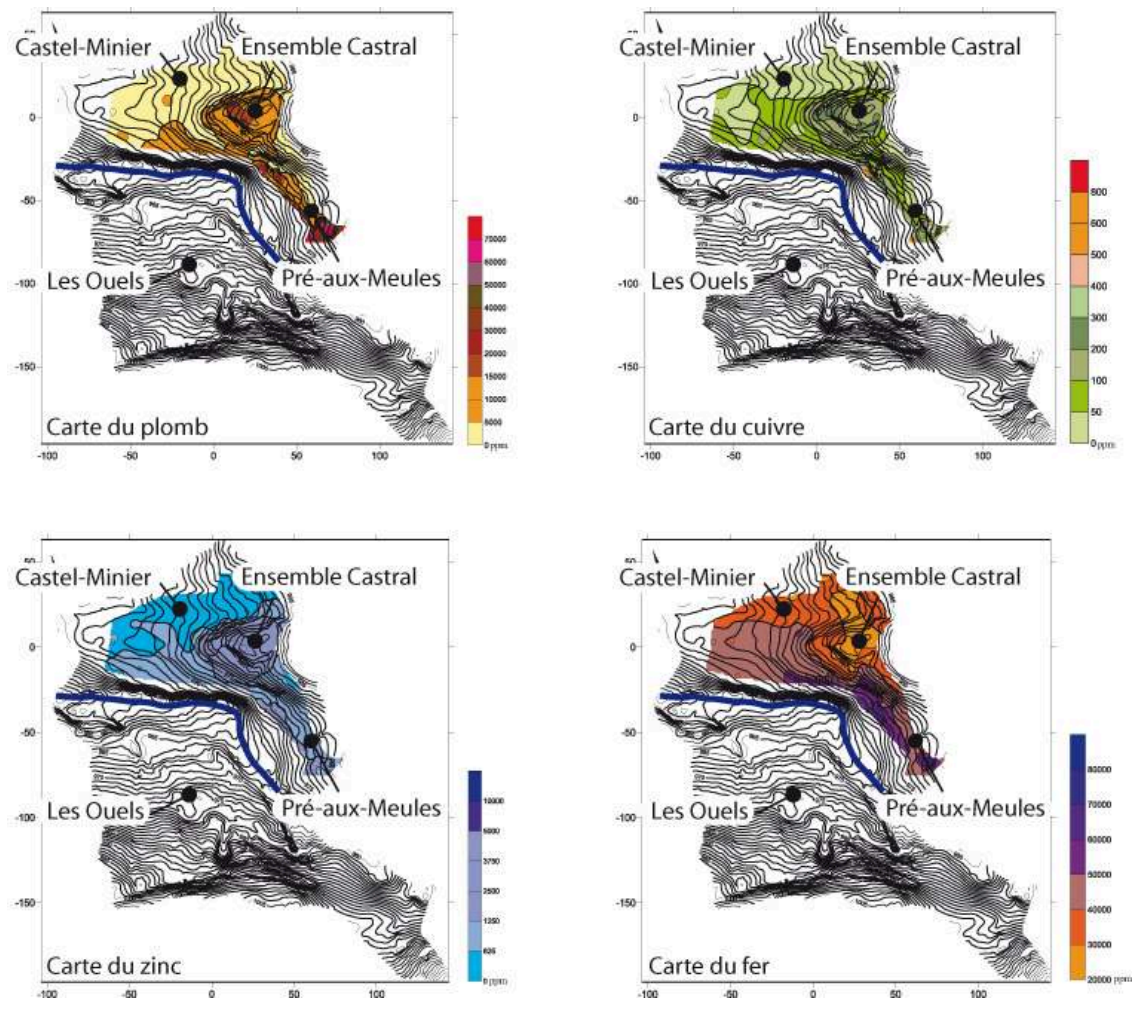

Les anomalies sur le bord du Garbet ainsi que dans le pré-aux-meules peuvent être interprétées à la lumière des informations historiographiques et archéologiques dont nous disposons: "[décrivant Castel-Minier et les travaux miniers]Il s'y trouvé tout aupres jusques à quatre-vingt-sept meules à moudre les mines.» (Malus [J. de], Recherche et découverte des mines des montagnes Pyrénées faites en l'an mille six cents par Jean de Malus et rédigée par J. du Puy, Toulouse, réédition, 1990.)

Lors de nos prospections et des fouilles qui s'en sont suivies, nous avons localisé 14 meules et fragments sur, ou à proximité de, ces deux zones. Le texte de Malus, le toponyme rappelant la présence de meules alors qu'aucune n'est visible actuellement dans ce pré et les trouvailles archéologiques forment avec les données géochimiques un faisceau d'indices conduisant à caractériser ces deux zones comme un lieu d'activité minéralurgique. Cette hypothèse est renforcée par le croisement, a priori, paradoxal des dosages du fer et du plomb (fig. 3). Dans une approche strictement relative, les taux les plus importants de fer se localisent en bordure du ravin et sur le pré-aux-meules. Or le fer est présent dans la minéralisation traitée mais va être évacué principalement lors des enrichissements du minerai mais également pendant la métallurgie extractive. En revanche, lors de l'affinage, seul du plomb d'œuvre est traité ne pouvant induire un enrichissement en fer des sols. Ainsi, sur la zone castrale, la corrélation entre les données géochimiques et historiques laissait entrevoir la mise au jour de la fonderie d'argent : « En la vicomté de Couserans à une lieue par-dessus le village d'Aulus il y a un château vieil, [...] il y a une vieille porte par laquelle on entrait dans la grande fonte, où l'on fondait l'or et l'argent. Ce château est appelé par ceux du pays le Castel-Minié.» 
(Malus [J. de], Recherche et découverte des mines des montagnes Pyrénées faites en l'an mille six cents par Jean de Malus et rédigée par J. du Puy, Toulouse, réédition, 1990.)

La destruction particulièrement bien soignée du château au tournant du XIX siècle n'a laissé que des traces somme toute ténues d'une activité métallurgique qu'il faut encore considérer comme hypothétique. La fouille de cet espace a néanmoins permis l'identification de la source de l'anomalie géochimique. Lors de l'édification de ce castrum, les bâtisseurs ont utilisé pour leur mortier des résidus de la production de l'argent (sable de lavage, scories broyées) en lieu et place de l'habituel sable. Si la source de l'anomalie en plomb repérée en prospection est identifiée, pour autant, nous ne pouvons exclure la présence de la fonderie sur ce lieu pour deux raisons (fig. 3). Tout d'abord, de nouveaux dosages ont été effectués sur les rares sols retrouvés en place. Les taux de plomb sont encore très élevés atteignant 20000 ppm sur l'unique niveau de sol mis au jour. D'autre part à l'occasion de la calibration de nos analyses, nous avons eu l'opportunité de doser l'argent. Cet élément, caractéristique de la dernière phase de production, apparaît avec des teneurs moyennes de 100 ppm alors que dans les zones où aucune activité métallurgie non-ferreuse n'est suspectée, ce taux est deux fois inférieur.

\section{Le cas d'Agnesserre}

Dans ce cirque de fond de vallée, des meules à minerai usagées ont été reconnues de longue date. La distance qui sépare cette zone de celle de Castel-Minier, plus de deux kilomètres, rend très hypothétique le déplacement vers l'amont de ces objets. Il est plus raisonnable de penser que des installations minéralurgiques ont existé dans ce lieu ouvert bénéficiant d'un flux hydrique plus facilement canalisable que dans la partie aval où le torrent se trouve encaissé dans une gorge étroite. Devant la surface à couvrir (près de 5 hectares), la nature du terrain encombrée de végétaux et le réseau hydrographique digité du Garbet à cet endroit, il a été choisi de prospecter par magnétisme avant d'entamer l'approche géochimique du lieu. 
Figure 4 : Carte magnétique du fond du cirque d'Agnesserre. Figure 4: Magnetic map of Agnesserre corrie.

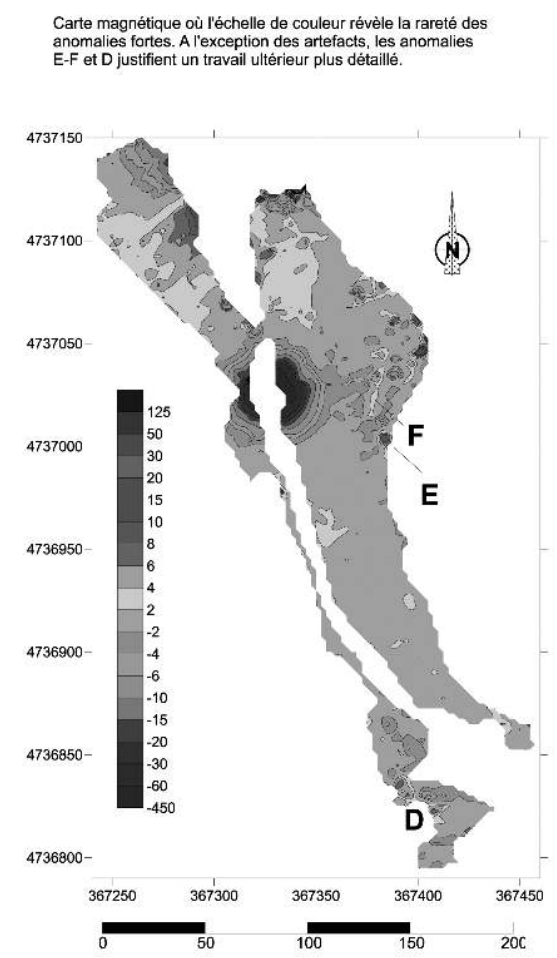

La prospection magnétique

Les contraintes identifiées pour cette zone ont impliqué la mise en place d'une stratégie de collecte d'informations peu usitée lors de prospections magnétiques sur site archéologique. Nous avons procédé en fixant un GPS de poche (Garmin 60CSx) sur le contrepoids du magnétomètre. Il permet le positionnement des mesures bien que le géoréférencement absolu soit médiocre (5 à $10 \mathrm{~m}$ RMS), l'usage du mode "track" assure une localisation relative correcte. Ce système conduit à avoir, sur la durée de quelques heures, une bonne cohérence « interne » de la carte géophysique ${ }^{6}$.

Avec le GPS fixé au contrepoids du magnétomètre, quelques corrections aux mesures sont nécessaires. La plus évidente concerne la position: le GPS est $1,5 \mathrm{~m}$ derrière le capteur magnétique et le sens de progression évolue au grès des allers-retours et des contraintes topographiques. Le magnétomètre et le GPS produisent un biais dépendant de l'orientation de l'ensemble. Nous avons mesuré ce biais qui atteint 1,8 nTesla pour le capteur du haut (utilisé pour le champ total 7 ). L'effet est maximum lorsque l'on regarde vers le nord, ce qui est compatible avec une hypothèse d'effet magnétique induit plutôt que rémanent. Avec le mode «track» du GPS, on dispose, toutes les secondes (par exemple), des points successifs, et donc de l'orientation du magnétomètre. Un programme de correction a été élaboré. Il effectue à la fois les corrections de directions et de positionnement. En testant «sans » ou «avec » ces corrections, nous avons noté une excellente amélioration de la qualité de la carte, ce qui confirme également la qualité de la localisation points GPS en mode relatif.

Cette première approche des réponses magnétiques du sous-sol reste stratégique. Elle est en mesure de détecter les zones minéralurgiques, mais sans en donner la position absolue à $5 \mathrm{~m}$ près. 
Sur la carte interprétative trois anomalies ressortent ${ }^{8}$ (fig. 4). Il est impossible d'en déterminer la source sans sondage, néanmoins on voit que la prospection magnétique conduit à drastiquement réduire la zone d'intérêt. Sur le terrain, l'anomalie $\mathrm{F}$ est proche $\mathrm{du}$ bas de pente, mais rien ne se distingue particulièrement. L'anomalie $\mathrm{E}$ se détache mieux. Topographiquement, elle s'inscrit par endroit dans un petit lit secondaire du torrent, comme il en existe d'autres sur le terrain qui ne produisent aucune anomalie. Enfin, au Sud de cette carte, deux anomalies (D) assez fortes se détachent. La confrontation avec une autre approche prospective comme la géochimie s'impose naturellement.

Figure 5 : Carte de la teneur en plomb du cirque d'Agnesserre.

Figure 5: Lead concentration map of Agnesserre corrie.

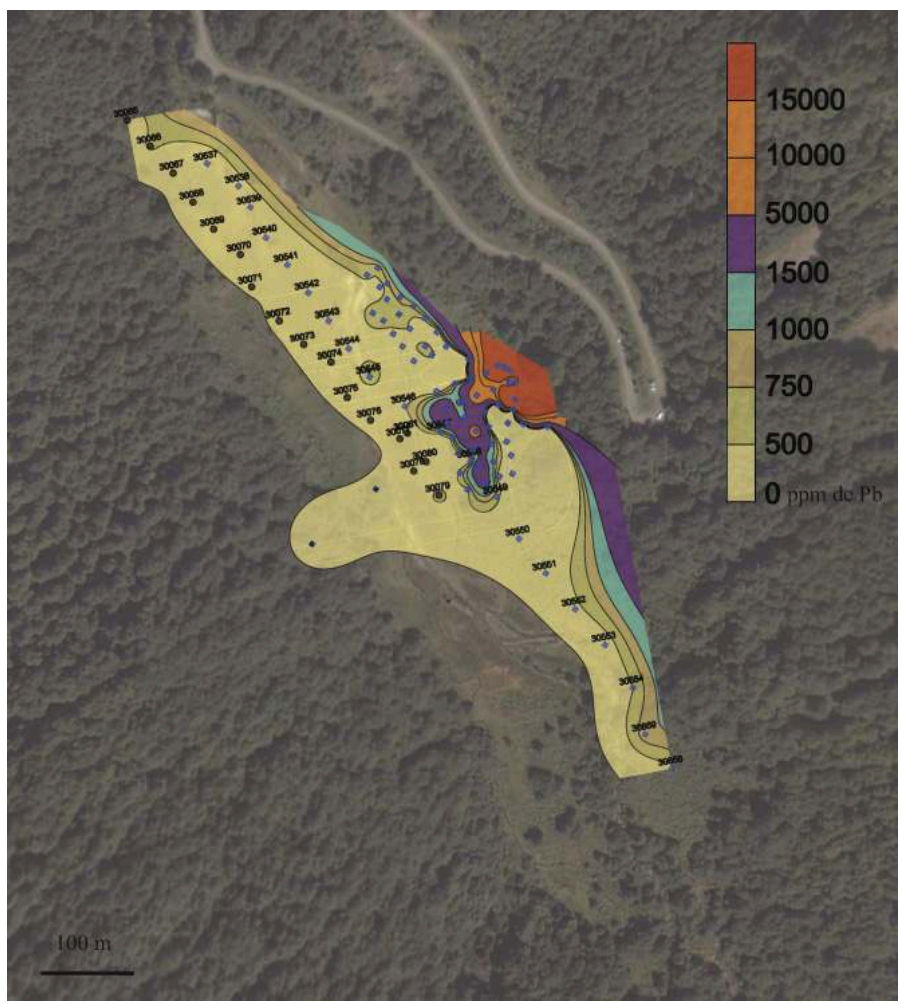

La prospection géochimique

Étant dans une démarche méthodologique, la mise en place de la prospection géochimique a suivi la même approche stratégique alors que, fort de la carte magnétique, il était envisageable de focaliser nos recherches sur les anomalies précédemment définies (fig.5). Dans un premier temps deux lignes parallèles de prélèvements dont une traverse longitudinalement la totalité du cirque d'Agnesserre, ont été définies regroupant près de 40 échantillons. Pour ces deux lignes, seuls les points 30547 à 30549 montrent une concentration légèrement supérieure à celles observées sur les autres échantillons. Un axe de prélèvement perpendiculaire a été défini permettant d'orienter rapidement la prospection sur le versant nord-est en lieu et place de l'anomalie géophysique $\mathrm{F}$. Une fois définie la zone d'intérêt, le mode de prélèvement prend un caractère intensif afin d'être à même de dresser une carte géochimique de l'espace couvert. À l'issue de 54 dosages supplémentaires, la carte qu'il est possible d'établir montre une brutale élévation de la concentration de plomb qui atteint, au milieu du cirque 1500 à 5000 ppm. Ce niveau gagne encore en intensité 
lorsque l'on arrive au contact de la pente nord-est (10 000 à 15000 ppm de plomb). Selon les résultats tant géochimiques que géophysiques, il semble même que nous ayons détecté deux événements distincts. Le plus faible, au centre du cirque, couvre une surface de $3000 \mathrm{~m}^{2}$. Le plus intense n'excède pas $2000 \mathrm{~m}^{2}$ et apparaît guidé par un ru. Est-ce un hasard? Mais deux des quatre meules connues dans ce cirque se trouvent sur cette zone comportant de fortes concentrations de plomb. Enfin, à l'occasion d'un des carottages dans l'espace le plus riche en plomb, nous avons observé la présence de petites scories (infra centimétriques). En fonction des données tant archéologiques qu'archéométriques, il est fort possible que nous ayons trouvé sinon l'emplacement d'un moulin à scorie, du moins la zone de rejet liée à cette activité. La présence de scories ne doit pas nous égarer. Bien que leur formation résulte d'une activité de réduction proprement métallurgique, leur taille liée aux autres indices dont nous disposons, les assimile bien à des résidus de traitement mécanique par broyage visant la récupération des billes de plomb?

\section{Étude stratigraphique de la pollution au plomb}

Dans son versant industriel, le site de Castel-Minier se décompose entre une activité de production d'argent et une production d'acier (Dillmann et al., 2006). Il apparaît que ces deux activités ne sont pas nécessairement synchrones. Spatialement, dans l'état de nos connaissances, l'activité touchant à la production du plomb et de l'argent est séparée de celle liée à la production du fer.

Les accumulations constitutives du ferrier de la mouline ont fourni une image complète de la phase d'activité sidérurgique et facilement accessible puisqu'en cours de fouille. Nous avons alors cherché à caractériser les taux de plomb dans le ferrier à partir des stratigraphies pour déterminer les couches correspondants à une activité synchrone des deux métallurgies de celles qui ne révèlent que l'activité liée au fer.

Figure 6 : liste des dosages par spectrométrie de fluorescence $X$ des US du ferrier de la mouline (présentées dans leur ordre chrono stratigraphique).

Figure 6: list of quantities determination by portable $X$ ray fluorescence on strata from mouline slag heap (given by chronological order).

\begin{tabular}{|r|l|r|r|r|}
\hline $\mathbf{N}^{\circ}$ d'analyse & Unité stratigraphique & Pb en ppm & Zn en ppm & Cu en ppm \\
\hline 146 & us 2001 & 810 & 890 & nd \\
\hline 135 & us 2001 & 2330 & 690 & 60 \\
\hline 150 & us 2075 & 1740 & 850 & 95 \\
\hline 153 & us 2088 & 1280 & 990 & 200 \\
\hline 136 & us 2069 & 2060 & 850 & 70 \\
\hline 140 & us 2071 & 2000 & 640 & nd \\
\hline 148 & us 2074 & 2050 & 620 & 70 \\
\hline 137 & us 2070 & 2890 & 390 & 80 \\
\hline 151 & us 2120 & 3590 & 570 & 30 \\
\hline 143 & us 2026 & 2050 & 370 & 45 \\
\hline 129 & us 2026 & 2420 & 490 & 10 \\
\hline 145 & us 2085 & 4070 & 470 & nd \\
\hline 124 & us 2084 & 12150 & 1330 & nd \\
\hline
\end{tabular}

Lors de la cartographie géochimique générale du site, la zone du ferrier a donné une réponse en plomb qui n'a jamais excédé 1000 ppm. Nous considérons, pour conserver 
une marge de sécurité large, que jusqu'à 3000 ppm, nous sommes dans des taux normaux de pollution pour ce site. Ils ne traduisent alors que le milieu ambiant dans lequel s'est déroulé l'activité. À cette aune, trois unités stratigraphiques (US) sortent alors du lot: les US 2120, 2085 et 2084. L'unité 2120 se présente comme fort en remblai contre le mur de la forge, les US 2085 et 2084 pour leur part sont des niveaux de sol mis au jour sous le ferrier. Un séquençage simple de l'activité sidérurgique semble bien se dégager traduisant la perduration de l'activité sidérurgique après l'abandon de l'activité minière et métallurgique non ferreuse. L'analyse géochimique confirme les données archéologiques qui montraient au tournant $d u \mathrm{xv}^{\mathrm{e}}$ siècle un abandon du château servant alors de carrière pour la rénovation du canal de la forge.

Le site aurait donc connu un glissement fonctionnel en trois temps allant d'une production unique de métal blanc vers la production concomitante du fer, du plomb et de l'argent et s'achevant par une unique activité sidérurgique. Il apparaît que l'abandon de la partie castrale traduit parallèlement l'intérêt porté à la production d'argent, ce qui ne faisait pas de doute, mais également la moindre attention donnée à la production du fer puisque le contrôle seigneurial disparait du site. Ce schéma méritera bien sûr d'être affiné. L'approche comparée de la stratigraphie et de la géochimie semble être un bon positionnement pour bien apprécier ce séquençage lors de la poursuite des fouilles archéologiques de Castel-Minier.

\section{Conclusions}

Ce travail ouvre de nouvelles perspectives pour la recherche des sites paléométallurgiques. Les résultats obtenus lors de notre propre calibration montrent la fiabilité des données recueillies sur le terrain à l'aide de cet appareillage pour les éléments caractéristiques des activités recherchées. Et quand bien même les données quantitatives ne seraient pas absolument exactes, l'approche statistique de cette prospection géochimique permet de palier aux erreurs de dosage. En effet, ce n'est pas tant une donnée absolue qui est recherchée qu'une image relative des éléments métalliques contenus dans les sols à l'échelle du site archéologique. Toujours à ce niveau d'observation, la prospection géochimique par spectrométrie de fluorescence $\mathrm{X}$ portable permet une localisation assez fine des espaces devant être sondés. L'exemple de la zone castrale, bien qu'il ne soit pas totalement positif au regard des vestiges mis au jour, comparé à celui du pré-aux-meules laisse envisager une possible distinction entre les lieux minéralurgiques et les ateliers métallurgiques. L'usage de cette méthode d'analyse en cours de fouille gagnera à être développé. Le cas de Castel-Minier regroupant une double production métallique peut être atypique mais le dosage des niveaux de sol des différents espaces mis au jour devrait faciliter, ici comme sur d'autres ateliers, la détermination des zones de travail.

Dans le cadre d'une prospection extensive, comme dans le cirque d'Agnesserre, la souplesse d'utilisation permet de couvrir des espaces importants dont la seule limite vient non plus du traitement analytique de l'échantillon mais de son relèvement. En effet la méthodologique mise en œuvre découle de la prospection par stream sediments. Mais elle ne cherche pas pour autant à étudier un bassin-versant. Son périmètre d'action est plus réduit et nécessite donc un recalage topographique optimal pour être porteuse d'information archéologique. 


\section{BIBLIOGRAPHIE}

Arrhenius, A., 1931. Die Bodenanalyse im Dienst der Archäologie, Zeitschrift für Pflanzenernährung, Düngung und Bodenkunde, Teil B, 10 Jahrgang, p. 427-439.

Bailly-Maître, M.-C. et Jacob, N., 2001. Analyse de la pollution minière de rivières de l'Oisans, application à l'archéologie, Archéologie du Midi médiéval, vol. 19, p. 67-77.

Berna, F., Behar, A., Shahack-Gross, R., Berg, J., Boaretto, E., Gilboa, A., Sharon, I., Shalev, S., Shilstein, S., Yahalom-Mack, N., Zorn, J. R. et Weiner, S., 2007. Sediments exposed to high temperatures: reconstructing pyrotechnological processes in Late Bronze and Iron Age Strata at Tel Dor (Israel), Journal of Archaeological Science, vol. 34, n³, p. 358-373.

Bonnamour, G., Florsch, N. et Téreygeol, F., 2007. Les prospections de ferriers de Castel-Minier : approches interdisciplinaires, ArchéoSciences, revue Archéométrie, n 31, p. 37-44.

Dillmann, P., Téreygeol, F. et Verna, C., 2006. Premières analyses métallographiques des produits sidérurgiques trouvés sur le site médiéval de Castel-Minier (Aulus-les-Bains, 09), Archéosciences Revue d'Archéométrie, $\mathrm{n}^{\circ}$ 30, p. 7-14.

Domergue, Cl. et Leroy, M., 2000. Mines et métallurgie en Gaule, recherches récentes, Gallia, $\mathrm{n}^{\circ} 57$, p. 1-158.

Helmig, D., Jackwerth, E. et Hauptmann, A., 1989. Archaeometallurgical fieldwork and the use of a portable X-ray spectrometer, Archaeometry, vol. 31, n² 2, p. 181-191.

Leroy, B., 1972. Théorie monétaire et extraction minière en Navarre vers 1340, Revue de Numismatique, p. 105-123.

Morasz, J.-G. et Pulou, R., 1997.Les meules rotatives des mines romaines de Villefranche-deRouergue (Aveyron) : La preuve chimique de leur usage « industriel », Revue d'archéométrie, $\mathrm{n}^{\circ} 21$, p. $141-144$

Nieminen, T. M., Ukonmaanaho, L. et Shotyk, W., 2002.Enrichment of $\mathrm{Cu}, \mathrm{Ni}, \mathrm{Zn}, \mathrm{Pb}$ and $\mathrm{As}$ in an ombrotrophic peat bog near a Cu-Ni smelter in Southwest Finland, The Science of The Total Environment, vol. 292, $\mathrm{n}^{\circ}$ 1-2, p. 81-89.

Ploquin, A., Allée, P., Bailly-Maître, M.-C., Baron, S., De Beaulieu, J.-L., Carignan, J., Laurent, S., Lavoie, M., Mahé-Le Carlier, C., Peytavin, J. et Pulido, M., 2003.Medieval lead smelting on the Mont-Lozère, southern France, in Archaeometallurgy in Europe, Milan, Italie, 2 t., t. 1, p. 635-644. 
Shalev, S., Shilstein, S. S. et Yekutieli, Y., 2006. XRF study of archaeological and metallurgical material from an ancient copper-smelting site near Ein-Yahav, Israel, Talanta, vol. 70, $\mathrm{n}^{\circ}$, p. 909-913.

Téreygeol, F., Gratuze B., Foy D. et Lancelot J., 2004.Les scories de plomb argentifères : une source d'innovation technique carolingienne? Artisans, industrie, Nouvelles révolutions du Moyen Âge à nos jours, Société Française d'Histoire des Sciences et des Techniques, Cahier d'histoire et de philosophie des sciences, $\mathrm{n}^{\circ}$ 52, p. 31-40.

Téreygeol, F., Arles, A. et Vivier, M.-C., 2007.La grotte de La Catette : recherches sur un atelier de fauxmonnayeurs, Document Final de Synthèse, Service Régional de l'Archéologie Languedoc-

Roussillon.

Wagner, G. A., Pernicka, E., Seeliger, T. C., Öztunali, Ö. et Baranyi, I., 1983. Geologische Untersuchungen zur fruhen Metallurgie in NW-Anatolien, Bulletin of the Mineral Research \& Exploration, Turkey, vol. 101, p. 47-81.

\section{NOTES}

1. Phénomène physique de déplacement de chaleur en présence d'un courant électrique au contact de deux matériaux différents.

2. Les masses des appareils transportables sont de l'ordre de 20 à $50 \mathrm{~kg}$.

3. Une vérification de la taille du spot a été réalisée en laboratoire à l'aide d'une plaque photosensible. La taille du spot est de $22,5 \mathrm{~mm}$ par $11 \mathrm{~mm}$ avec une intensité maximale dans la moitié inférieure de la fenêtre.

4. En collaboration avec le laboratoire CEMHTI.

5. Parmi ces éléments, nous avons particulièrement observé les dosages obtenus pour $\mathrm{Hg}, \mathrm{Ag}, \mathrm{Zn}$, $\mathrm{Sn}, \mathrm{Sb}, \mathrm{Pb}, \mathrm{Cu}$ et $\mathrm{As}$.

6. L'idéal eût été de disposer d'un GPS différentiel - temps-réel ou non. Il n'est pas certain qu'un système différentiel fonctionne bien sur cette zone, du fait des masques très importants que constituent les montagnes alentours.

7. Nous avons regardé le champ total (corrigé de la dérive grâce à un magnétomètre à proton G856 mis en «base »), ainsi que le gradient. L'analyse géostatistique des variogrammes montre que le champ total est alors bien échantillonné, tandis que ce n'est pas le cas pour le gradient. Il n'y a pas de surprise à cela : l'essentiel est de savoir que les mesures magnétiques obtenues dans les conditions décrites sont bien significatives et fiables.

8. Les autres anomalies notées $\mathrm{A}, \mathrm{B}$ et $\mathrm{C}$ ont pu rapidement être écartées, leurs origines étant connues et actuelles.

9. Le retraitement de la scorie de la métallurgie des non-ferreux, s'il est encore mal perçu pour la période médiévale, semble incontournable (Téreygeol F. et al., 2004.).

\section{RÉSUMÉS}

La difficulté liée à la détection des ateliers de métallurgie des non-ferreux a conduit à tester un nouveau mode de prospection par spectrométrie de fluorescence X portable. Après une phase de 
calibration qui a donné de très bons résultats pour les métaux recherchés dans cette étude, trois approches ont été tentées sur le site de Castel-Minier (site minier et pluri métallurgique du second Moyen Âge, Ariège, France). Grâce à un travail interdisciplinaire incluant à la géochimie, l'archéologie, l'histoire et la géophysique, les résultats ont été particulièrement positifs. Ils ont conduit à une meilleure perception chronologique du site, à la localisation d'ateliers de traitement de scorie et la sériation des anomalies géochimiques.

Difficulties related to non-ferrous metal workshops detection led to test a new way of survey by a real portable x-ray spectrometer. After a calibration phase which yielded very good results for lead, zinc and cooper, three approaches were tried in south-west of France at the site of CastelMinier, (mining and multi metallurgical site from the second Middle Ages). Thanks to an interdisciplinary work that include geochemistry, archeology, history and geophysic, the results were particularly positive. They led to a better understanding of chronological phases. They give the location of slag processing workshops and classification of geochemical anomalies.

\section{INDEX}

Keywords : extensive survey, intensive survey, geochemistry, X-Ray fluorescence spectrometry, lead

Mots-clés : prospection extensive, prospection intensive, spectrométrie de fluorescence X, plomb

\section{AUTEURS}

FLORIAN TÉREYGEOL

UMR 5060 IRAMAT-LMC, Belfort et UMR 3299 SIS2M-LAPA, CEA - Saclay.

\section{A. ARLES}

UMR 5060 IRAMAT-CEB, Orléans.

E. FOY

UMR 3299 SIS2M-LAPA, CEA - Saclay.

NICOLAS FLORSCH

IRD UR 079 GEODES et UMR 7619 UPMC, Paris.

\section{LLUBES}

UMR5566 LEGOS, Toulouse. 